\title{
Association between cognitive performance, physical fitness, and physical activity level in women with chronic fatigue syndrome
}

\begin{abstract}
Kelly Ickmans, PT, MSc; ${ }^{1-3}$ Peter Clarys, PE, BR Sc, PhD; ${ }^{4}$ Jo Nijs, PT, PhD; $;^{1-3^{*}}$ Mira Meeus, PT, PhD; ${ }^{1-2,5}$ Dirk Aerenhouts, PE, PhD; ${ }^{4}$ Evert Zinzen, PE, PT, PhD; ${ }^{6}$ Senne Aelbrecht, PE; ${ }^{1,6}$ Geert Meersdom, MN; ${ }^{7}$ Luc Lambrecht, MD, PhD; ${ }^{8}$ Nathalie Pattyn, MD, PhD $^{\mathbf{9}}$

${ }^{1}$ Pain in Motion Research Group (PIM), Department of Human Physiology, Vrije Universiteit Brussel, Brussels, Belgium; ${ }^{2}$ PIM, Division of Musculoskeletal Physiotherapy, Department of Health Care Sciences, Artesis University College Antwerp, Antwerpen, Belgium; ${ }^{3}$ PIM, Department of Physical Medicine and Physiotherapy, University Hospital Brussels, Brussels, Belgium; ${ }^{4}$ Department of Human Biometry and Biomechanics, Vrije Universiteit Brussel, Brussels, Belgium; ${ }^{5}$ Department of Rehabilitation Sciences and Physiotherapy, Ghent University, Ghent, Belgium; ${ }^{6}$ Department of Sports Training and Movement Education, Vrije Universiteit Brussel, Brussels, Belgium; ${ }^{7}$ CVS Contactgroep VZW, Brugge, Belgium; ${ }^{8}$ Private practice for internal medicine, Ghent/Aalst, Belgium; ${ }^{9}$ Department of Human Physiology, Vrije Universiteit Brussel, Brussels, Belgium; and VIPER Research Unit, Royal Military Academy, Brussels, Belgium
\end{abstract}

\begin{abstract}
Limited scientific evidence suggests that physical activity is directly related to cognitive performance in patients with chronic fatigue syndrome (CFS). To date, no other study has examined the direct relationship between cognitive performance and physical fitness in these patients. This study examined whether cognitive performance and physical fitness are associated in female patients with CFS and investigated the association between cognitive performance and physical activity level (PAL) in the same study sample. We hypothesized that patients who performed better on cognitive tasks would show increased PALs and better performance on physical tests. The study included 31 women with CFS and 13 healthy inactive women. Participants first completed three cognitive tests. Afterward, they undertook a test to determine their maximal handgrip strength, performed a bicycle ergometer test, and were provided with an activity monitor. In patients with CFS, lower peak oxygen uptake and peak heart rate were associated with slower psychomotor speed $(p<0.05)$. Maximal handgrip strength was correlated with working memory performance $(p<0.05)$. Both choice and simple reaction time were lower in patients with CFS relative to healthy controls ( $p<0.05$ and $p<0.001$, respectively). In conclusion, physical fitness, but not PAL, is associated with cognitive performance in female patients with CFS.
\end{abstract}

Key words: aerobic capacity, chronic fatigue syndrome, cognitive functioning, maximal handgrip strength, physical activity, physical fitness, psychomotor speed, reaction time, vigilance, working memory.
Abbreviations: ANOVA = analysis of variance, $\mathrm{CDC}=\mathrm{U} . \mathrm{S}$. Centers for Disease Control and Prevention, CFS = chronic fatigue syndrome, $\mathrm{HR}=$ heart rate, $\mathrm{HR}_{\mathrm{p}}=$ peak heart rate, $\mathrm{HR}_{\text {rest }}=$ resting heart rate, MET = metabolic equivalent, OSPAN = operation span task, PAL = physical activity level, $\mathrm{PVT}=$ psychomotor vigilance task, $\mathrm{RER}_{\mathrm{p}}=$ peak respiratory exchange ratio, $\mathrm{SD}=$ standard deviation, $\mathrm{SWA}=$ SenseWear $^{\circledR}$ Pro3 Armband, $\mathrm{VO}_{2 \mathrm{p}}=$ peak oxygen uptake.

*Address all correspondence to Jo Nijs, PT, PhD; Vrije Universiteit Brussel, Faculty of Physical Education and Physiotherapy, Department of Human Physiology, Building L, Pleinlaan 2, 1050 Brussels, Belgium; +32-2-6291154; fax: +32-2-6292876. Email: Jo.Nijs@vub.ac.be http://dx.doi.org/10.1682/JRRD.2012.08.0156 


\section{INTRODUCTION}

Chronic fatigue syndrome (CFS) is defined by the U.S. Centers for Disease Control and Prevention (CDC) as a condition characterized by self-reported unexplained persistent or relapsing fatigue of at least 6 mo duration and the concurrent occurrence of multiple nonspecific symptoms, including sore throat, muscle and joint pain, headache, tender cervical or axillary lymph nodes, unrefreshing sleep, and postexertional malaise [1].

Cognitive complaints are some of the most frequent and significant contributors to social and occupational dysfunction in people with CFS [2]. These complaints are particularly mentioned in the form of memory and concentration problems [3-4]. Some studies, in which objective measures were used to evaluate cognitive performance, have reported slowed psychomotor and/or processing speed [5-10], impaired working memory [6-8,11-12], and poor information learning [3,8,13-15] in patients with CFS, while others found no abnormalities in these domains (psychomotor and/or processing speed [11,1617], working memory [3,18], and information learning $[16,19])$. Differences in testing batteries, diagnostic heterogeneity, comorbid psychiatric disorders, and medication usage could be the cause of these inconsistent findings.

Besides the frequently reported cognitive complaints in people with CFS, daily physical activity level (PAL), measured by accelerometry (counts/time), is also significantly reduced relative to healthy controls. PAL refers to the amount of physical activity performed during daily life. This was confirmed by the results of two systematic literature reviews [20-21]. In both reviews [20-21], all the included studies on PAL in patients with CFS ( $n=5$ in both reviews) used real-time accelerometry and found significantly lower PALs among patients with CFS than in healthy controls. Furthermore, PALs in people with CFS seem to correlate with symptom severity and variability [22]. In their discussion paper, Wessely et al. suggested that persistent inactivity caused by symptoms like pain and fatigue leads to deterioration of physiological exercise capacity and the subsequent presence of more symptoms, which eventually results in a vicious and selfperpetuating cycle of activity avoidance [23]. This avoidance behavior toward physical activity is in turn likely to influence PAL and exercise performance. A logical consequence would be that the exercise capacity in patients with CFS is significantly lower than in healthy controls. However, a systematic literature review recently con- ducted by Nijs et al. revealed no definite conclusion in relation to physiological exercise capacity in patients with CFS [20]. Sources of bias such as the lack of uniformity in sex, exercise testing protocols, PAL, and utilized diagnostic criteria for CFS might be explanatory factors for these conflicting findings.

In previous work, a significant positive correlation was found between cognitive dysfunction and low PALs [24] and cognitive impairment and the degree of functional impairment among patients with CFS [25]. These findings suggest that physical activity is directly related to cognitive performance in patients with CFS. Yet to date, no study has examined the direct relationship between cognitive performance and physical fitness (and physiological exercise capacity in particular) in people with CFS.

First, this study aimed at examining the relation between cognitive performance and physical fitness in female patients with CFS. Likewise, the relation between cognitive performance and PAL was examined. We hypothesized that patients who performed better on cognitive tasks would show increased PALs and better performance on physical tests.

Second, given the inconsistencies in available data both in terms of objective cognitive findings and physiological exercise capacity findings in CFS, this study examined cognitive performance and physical fitness in female patients with CFS. For this purpose, we excluded patients experiencing major depression with psychotic or melancholic features and/or other psychiatric disorders that can explain the presence of chronic fatigue from study participation. Furthermore, we compared the results of patients with CFS with those of an inactive sex-, age-, height-, and body mass-matched healthy control group.

\section{METHODS}

\section{Study Design}

The study was designed as a prospective case-controlled comparison and took place at a private practice for internal medicine in Ghent (Belgium).

\section{Participants}

Patients with CFS were recruited via a private practice for internal medicine. To be included in the study, they had to comply with the 1994 CDC criteria for CFS [1]. The same internal medicine physician diagnosed all 
patients. Other inclusion criteria included being female, because pooling of sex data has been identified as an important source of bias in addressing exercise physiology in patients with CFS [26], and between 18 and $45 \mathrm{yr}$ old. Based on the results of a previous study on cognitive performance in patients with CFS, the power analysis revealed that a sample size of 31 patients with CFS would provide an 80 percent power and alpha of 0.05 [8].

We asked all of the included patients with CFS to bring a healthy (pain-free and without any [chronic] disease) and inactive relative, friend, or acquaintance to participate in the control group. We defined inactive as having a seated occupation and performing a maximum of $1 \mathrm{~h}$ of exercise per week [27]. Controls had to meet the same additional inclusion criteria (sex and age) as the patients with CFS. Exclusion criteria for both the patients with CFS and controls included pregnancy and the presence of intellectual disabilities. The internal medicine physician carefully selected the patients with CFS and thereby excluded all women who were pregnant. This was asked in advance of both the patients and controls. In any case of doubt, a pregnancy test was used. To determine the presence of intellectual disabilities, we relied on the medical records of the patients with CFS. If the diagnosis was not mentioned in the medical record, we assumed that no intellectual disability was present. The controls had to be pain-free and could not suffer from any (chronic) disease. This was always asked in advance, and when the presence of a disease was suspected, the general practitioner could always be contacted. This was never necessary. Furthermore, on the testing day, we asked every participant to refrain from using substances like caffeine, alcohol, and nicotine, which could influence physical and/or cognitive performance. We excluded participants who used drugs known to influence cognitive function (opioids, antidepressants, anticonvulsants, benzodiazepines).

\section{Procedure}

Potential study participants were first informed about the study by their physician, then asked to read an information leaflet. Next, written informed consent was obtained from all participants. After we collected information on personal characteristics and measured participants' weight and height, we asked them to complete three cognitive tests. To examine physical fitness, all participants completed a test to determine their maximal handgrip strength, performed an exercise test on a bicycle ergometer with continuous cardiorespiratory monitoring (ergospirometry), and were provided with an activity monitor to register daily physical activity during 72 consecutive hours. Figure 1 shows the flowchart of the study.

\section{Cognitive Tests}

To investigate cognitive function, we used the Stroop task, the psychomotor vigilance task (PVT), and the operation span task (OSPAN) with concomitant mathematical processing to assess selective attention and choice reaction time, vigilance and alertness, and working memory capacity, respectively. All three tests were conducted on the same computer and in the same order (Stroop task, PVT, OSPAN) by every participant. To ensure standardization of the procedure, each test began with the presentation of written instructions for that particular test. Short breaks ( $\pm 5 \mathrm{~min}$ ) between each test were allowed.

In the Stroop task [28] examining cognitive control, all the possible interference effects inherent to the Stroop paradigm were examined (semantic, emotional, and priming). In this task, responses were collected through the computer keyboard. Different words were presented one by one and in various font colors (yellow, green, red, and blue) in the middle of the screen on a white background. Participants were instructed to respond to the presented font color in which words were written by pressing the corresponding colored key as quickly and as accurately as possible. The computer stored response times and accuracy. The task began with a practice block,

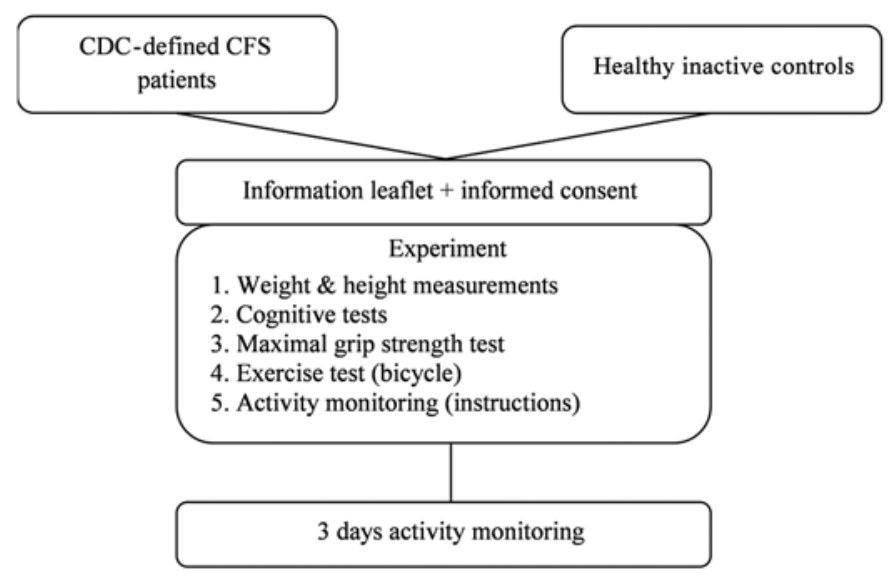

Figure 1.

Flowchart of study. CDC $=$ U.S. Centers for Disease Control and Prevention, CFS = chronic fatigue syndrome. 
during which participants received feedback about their performance ("correct" or "incorrect" and response time). The presented words/nouns could be classified under eight different conditions, namely category (animal names written in one color), congruent (same word and color, e.g., the word "red" displayed in red font), incongruent (different word and color, e.g., the word "red" displayed in green font), neutral (neutral words written in one color, e.g., “windowsill," "plate," “circus”), no word ("XXX" presented in one color), priming negative inverse (e.g., the word "red" displayed in green font immediately followed by the word "green" displayed in red font), priming negative simple (e.g., the word "red" displayed in green font immediately followed by the word "blue" displayed in red font), and sleep (sleep-related words written in one color, e.g., "nap," "night[time]," "fatigue," "blanket”).

In the PVT [29], which is a test of simple reaction time, participants responded to a visual stimulus that appeared on the screen (always in the middle) at random interstimulus intervals (2-10 s). The written instructions told them to take the mouse in their dominant hand, not to support their arm, and to press either the left (for lefthanded people) or the right (for right-handed people) mouse button with the thumb as quickly as possible whenever they perceived the appearance of the stimulus (a fireball) on the screen. Participant's reaction time was displayed on the screen for $1 \mathrm{~s}$. If the participant did not respond within $500 \mathrm{~ms}$, the trial was stored as a lapse and a warning message appeared. The PVT test duration was $10 \mathrm{~min}$.

Like the PVT, the OSPAN (based on Conway and Engle [30]) was completely mouse-driven. The task began with a practice block (divided into three sections). First, participants practiced the simple letter span in which they saw letters appear on the screen one at a time and had to recall these letters in the same order they saw them. The second practice was the math portion of the OSPAN. First, a math operation appeared on the screen (e.g., $(7 \times 3)-3=$ ?). Next, a number (e.g., 18) was presented and participants were instructed to click if the number was the correct solution by clicking on "true" or "false." After the recall of each letter span and after each math operation, participants were given feedback about their performance. To account for individual differences, the program calculated how long it took a given participant to solve each of the math problems. The average time plus 2.5 standard deviation (SD) was then used in the experimental block as a time limit to solve the operations, thus preventing participants from rehearsing the letters when they should be solving the operations. The final practice session consisted of exercises as they would appear during the experimental block, meaning that both the letter recall and the math operations had to be performed together. Participants first had to solve the math operation and only then saw the letter to be recalled. After the completion of all practice sessions, the program automatically proceeded to the experimental block, which consisted of three sets of each set size (ranging from 3 to 7). Thus, a total of 75 letters and 75 math problems were presented. During letter recall, a math accuracy percentage was presented in the upper right-hand corner. Participants were instructed to keep their math accuracy at or above 85 percent at all times. At the end of the OSPAN experiment, five values were registered, namely score (sum of the letters in all perfectly recalled letter sets), total (sum of letters recalled in the correct position, regardless of whether the set was correct), accuracy error (number of wrong answered math operations), speed error (number of answers beyond the allowed time), and math error (sum of accuracy error and speed error).

\section{Maximal Handgrip Strength Test}

We measured maximal handgrip strength using a hydraulic hand dynamometer (Jamar dynamometer, Saehan Corporation; Masan, Japan) with an adjustable handle and an analogous reproduction of the delivered power in kilogram-force and pound-force. Participants sat on a chair while holding the dynamometer in their dominant hand with the elbow flexed at $90^{\circ}$ and the forearm in neutral position. The analog screen was turned away from the participants so they could not read the amount of generated force. They were then instructed to grip the instrument as hard as possible in three consecutive attempts. The highest score was recorded. Data from a study by Peolsson et al. demonstrated excellent inter- and intrarater reliability for the Jamar dynamometer with intraclass correlation coefficients ranging from 0.94 to 0.98 [31]. This type of hydraulic hand dynamometer also showed good concurrent validity $(r=0.9998)$ with known weights [32].

\section{Exercise Test}

Participants performed the exercise test on a computer-controlled cycle ergometer (eBike Comfort, GE 
Healthcare; Freiburg, Germany). They were first given 3 to 5 min to adjust to the test position, after which we collected baseline data. Pulmonary data (peak oxygen uptake $\left[\mathrm{VO}_{2 \mathrm{p}}\right]$ and peak respiratory exchange ratio $\left[\mathrm{RER}_{\mathrm{p}}\right]$ ) were acquired breath-by-breath using a computerized Vmax-229 series spirometer (SensorMedics; Yorba Linda, California). Heart rate (HR) was calculated using an electrocardiograph (Cardiosoft ${ }^{\mathrm{TM}}$, GE Healthcare). The test started at a workload of $60 \mathrm{~W}$, which was increased by $30 \mathrm{~W}$ every minute. Participants pedaled at a speed of 60 to 70 rotations per minute. The exercise test was finished when they were exhausted and, consequently, their pedaling rate sank below 55 rotations per minute. Cool-down included $1 \mathrm{~min}$ of cycling at a rate of 20 rotations per minute and a workload of $30 \mathrm{~W}$.

\section{Real-time Activity Monitoring}

We used the SenseWear ${ }^{\circledR}$ Pro3 Armband (SWA) (BodyMedia Inc; Pittsburgh, Pennsylvania) wireless multisensor accelerometer for real-time monitoring of physical activity of all participants during 3 consecutive days. This activity monitor has a two-axis accelerometer, along with several other physiological sensors (heat flux, skin temperature, near-body ambient temperature, body position, movements of the upper arm, and galvanic skin response) from which data are integrated and can subsequently be uploaded and analyzed using computer software. Energy expenditure, which we calculated at $1 \mathrm{~min}$ intervals for this study, is estimated based on sex, age, height, and weight, together with the information collected from all sensors. The SWA also registered the time when energy expenditure was $>3$ metabolic equivalents (METs), the time spent sleeping, and the time spent awake in supine position. The SWA is lightweight and comfortable to wear, worn on the back of the right upper arm over the triceps muscle. Good validity and reliability of the SWA has been shown in healthy adults under laboratory [33] and free-living conditions [34].

\section{Statistical Analysis}

We performed data analyses using SPSS version 19.0 for Windows (IBM Corporation; Armonk, New York). All data subsets were assessed for normal distribution using the Kolmogorov-Smirnov goodness of fit test and appropriate descriptive statistics were calculated. For normally distributed data subsets, parametric statistics were used (Student $t$-test, Pearson correlations). Subsets that were not normally distributed were analyzed with nonparametric tests (Mann-Whitney $U$ test, Spearman correlations). Stroop interference, priming, and emotional effects were investigated separately using a $3 \times 2$ analysis of variance (ANOVA), with Stroop condition as within-subject factor and group as between-subject factor. To investigate the Stroop interference effect, we performed the ANOVA on the mean reaction times of the congruent, incongruent, and no word conditions. Mean reaction times of incongruent, negative priming inverse, and negative priming simple stimuli were analyzed together in order to determine possible priming effects. The presence of emotional Stroop effects was investigated by performing an ANOVA on the mean reaction times of three conditions comprising nouns with different levels of emotional load, namely animal names (category condition), neutral words, and sleep-related words. After an arcsine transformation, Stroop accuracy data were also submitted to a $3 \times 2$ ANOVA and further analyzed in the same way as the Stroop reaction time data. The significance level for all statistical tests was set a priori at $p<0.05$.

\section{RESULTS}

We recruited 31 women with CFS. From all of the controls who agreed to study participation, we were able to recruit and match 13 with patients with CFS. The controls consisted of healthy women matched for age, height, body mass, and body mass index, as shown in Table 1. All participants had the same ethnic background (native Dutch-speaking women from Flanders). Because of missing data due to dropout (of the patients with CFS, 1 refused to perform the exercise and maximal handgrip strength test because she felt nauseous and 6 did not wear the SWA properly), data loss (1 participant in both groups for the maximal handgrip strength test), technical problems with the test computer (1 patient with CFS and 2 controls for the PVT and OSPAN and 1 control for the Stroop task), and technical problems with the SWA (3 patients with CFS), the number of data analyzed for each test in each group does not always equal the total number of recruited participants.

\section{Cognitive Performance: Group with Chronic Fatigue Syndrome Versus Healthy Inactive Control Group}

\section{Stroop Task}

The $3 \times 2$ ANOVA on Stroop reaction times of the congruent, incongruent, and no word conditions exhibited a significant main effect of condition $(F(2,82)=6.31, p<$ 
Table 1.

Baseline characteristics of patients with chronic fatigue syndrome (CFS) and healthy inactive control participants. Data presented as mean \pm standard deviation.

\begin{tabular}{lccc}
\hline Characteristic & CFS $(\boldsymbol{n}=\mathbf{3 1})$ & Control $(\boldsymbol{n}=\mathbf{1 3})$ & $\boldsymbol{p}^{\text {-Value }}$ \\
\hline Age $(\mathrm{yr})$ & $35.6 \pm 7.6$ & $29.0 \pm 12.0$ & 0.12 \\
Body Mass (kg) & $64.9 \pm 10.8$ & $64.6 \pm 9.5$ & 0.93 \\
Height (cm) & $166.5 \pm 5.1$ & $168.4 \pm 6.9$ & 0.36 \\
Body Mass Index & $23.4 \pm 3.7$ & $22.8 \pm 3.7$ & 0.68 \\
\hline${ }^{*}$ Cody
\end{tabular}

*Comparisons performed using Student $t$-test.

$0.01)$ and group $(F(1,41)=7.11, p=0,01)$ but no interaction between both $(F(2,82)=1.44, p>0.1)$. Subsequent post hoc analysis (Bonferroni correction) revealed that the differences between reaction times on the incongruent and congruent conditions as well as between the incongruent and no word conditions were significant $(p<0.05$ and $p=0.01$, respectively) (Figure 2(a)).

For reaction times of incongruent, negative priming inverse, and negative priming simple stimuli, the ANOVA showed a significant main group effect $(F(1,41)=$ $6.26, p<0.05)$. There was no effect of condition $(F(2,82)=$ $1.01, p>0.1)$ and no interaction between group and condition $(F(2,82)=1.95, p>0.1)($ Figure 2(b)).

Figure 2(c) depicts the significant difference in reaction times between the CFS and control groups for words with different levels of emotional load (main effect of group) $(F(1,41)=7.23, p=0.01)$. No significant effect of condition $(F(2,82)=0.51, p>0.1)$ or interaction between group and condition $(F(2,82)=0.35, p>0.1)$ was found.

For Stroop accuracy of the congruent, incongruent, and no word conditions, a $3 \times 2$ ANOVA showed a marginally significant main effect of condition $(F(2,82)=$ $3.1, p=0.05)$ but no effect of group $(F(1,41)=2.76, p>$ $0.1)$ and no interaction between group and condition $(F(2,82)=1.55, p>0.1)$. The post hoc analysis for condition revealed marginally significant differences between accuracy scores of the congruent and incongruent conditions $(p=0.05)$ (Figure 2(d)).

No effect of group $(F(1,41)=0.01, p>0.1)$, condition $(F(2,82)=1.33, p>0.1)$, or interaction $(F(2,82)=$ $0.2, p>0.1)$ was found for accuracy scores in priming tasks (incongruent, negative priming inverse, and negative priming simple) (Figure 2(e)).

Figure 2(f) depicts a significant main effect of group $(F(2,82)=3.2, p<0.05)$ and condition $(F(1,41)=4.15, p<$ $0.05)$ and an interaction effect between both $(F(2,82)=$ $5.75, p<0.01$ ) for Stroop accuracy scores in words with different levels of emotional load. The post hoc analysis for condition revealed a marginally significant difference ( $p=0.05$ ) between Stroop accuracy for animal names (category condition) and sleep-related words in the control group. Because we wanted to investigate whether the main effect of group was not only the result of the large visible difference (Figure 2(f)) in accuracy for sleeprelated words, we submitted the accuracy data of all three conditions to a Mann-Whitney $U$ test. The analyses revealed a significant difference between Stroop sleep condition accuracy scores $(p=0.01)$, with superior scores in the CFS group. No differences were found in Stroop category and neutral condition accuracy (both $p>0.1$ ).

Psychomotor Vigilance Task and Operation Span Task

Table 2 presents the mean scores of both groups on the PVT and OSPAN. Patients with CFS showed a significantly higher percentage of lapses on the PVT (mean \pm SD: $30.6 \% \pm 36.6 \%)$ than controls $(2.8 \% \pm 1.9 \%)(p<$ $0.001)$. No significant differences were found in any of the five OSPAN scores $(p>0.05)$.

\section{Physical Fitness: Group with Chronic Fatigue Syndrome Versus Healthy Inactive Control Group}

Data acquired during the bicycle ergometer exercise test revealed that patients with CFS achieved a significantly lower workload at peak effort and had a lower peak HR $\left(\mathrm{HR}_{\mathrm{p}}\right)$ and $\mathrm{VO}_{2 \mathrm{p}}(p<0.001)$ than controls. Resting $\mathrm{HR}\left(\mathrm{HR}_{\mathrm{rest}}\right), \mathrm{RER}_{\mathrm{p}}$, and maximal handgrip strength were not found to be different between groups $(p>0.05)$ (Table 3).

\section{Relationship Between Cognitive Performance and Physical Fitness in Patients with Chronic Fatigue Syndrome}

$\mathrm{VO}_{2 \mathrm{p}}$ was inversely related to all components of Stroop reaction time as well as to PVT reaction time ( $p<$ 0.05 ). The analyses also yielded significant negative correlations between five out of eight Stroop reaction time parameters and $\mathrm{HR}_{\mathrm{p}}$ and between reaction time 
(a)

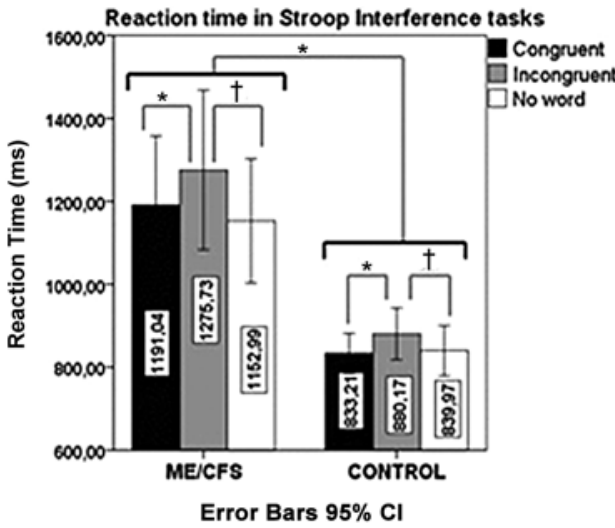

(c)

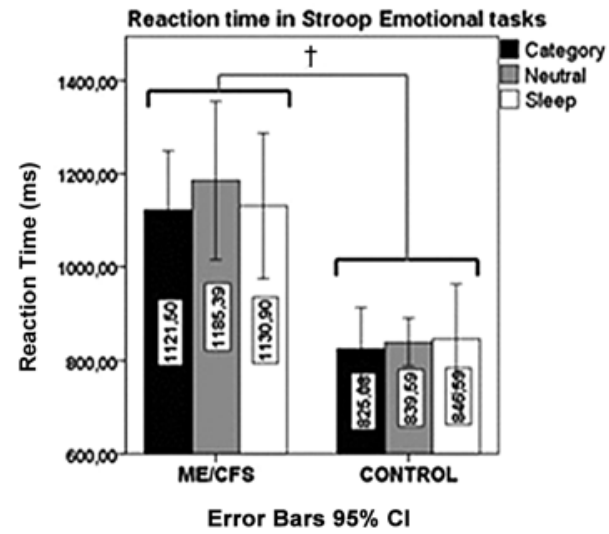

(e)

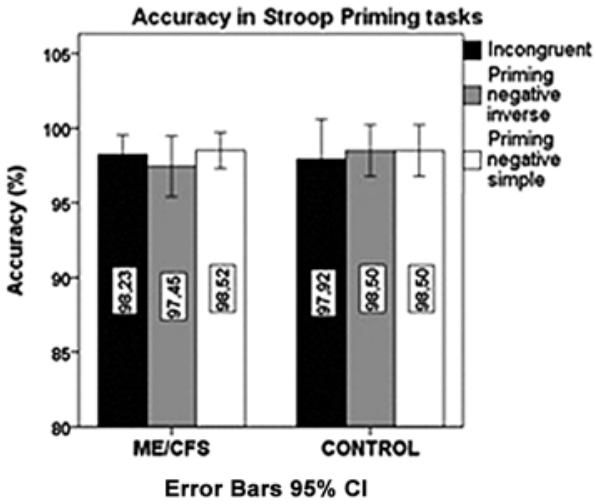

(b)

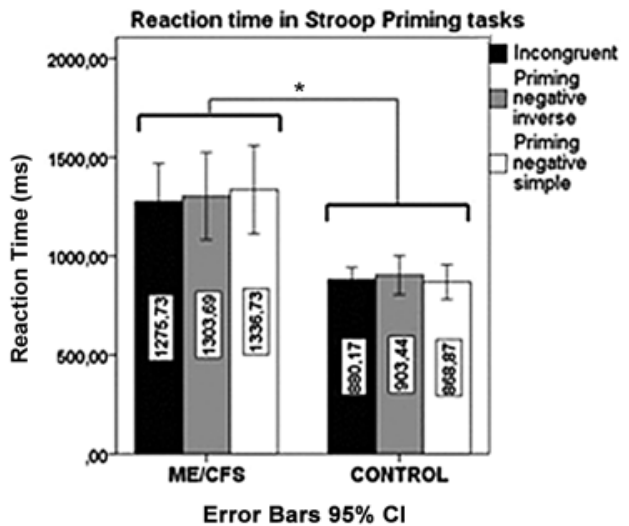

(d)

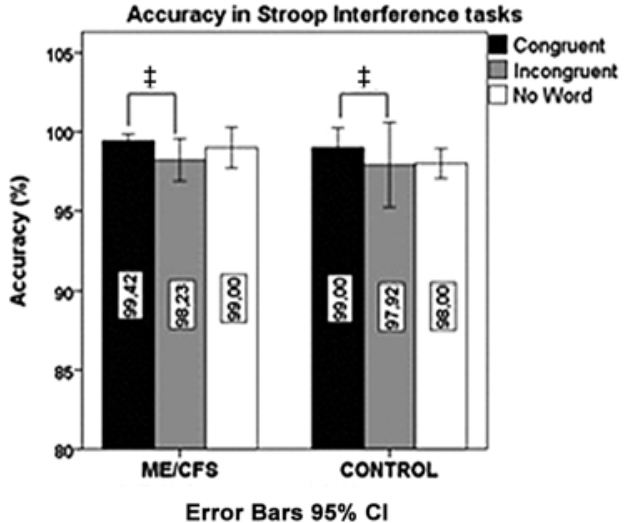

(f)

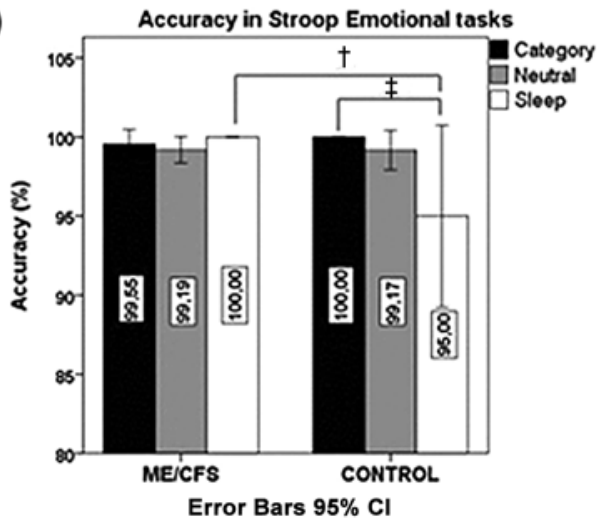

Figure 2.

Stroop task performance. (a) Difference in reaction times between patients with chronic fatigue syndrome (CFS) and healthy inactive control participants in congruent, incongruent, and no word conditions, and difference in reaction times in incongruent and congruent and in incongruent and no word conditions in both patients with CFS and controls indicating Stroop interference effect of same magnitude. Difference in reaction times between patients with CFS and controls in (b) priming tasks and (c) nouns with different levels of emotional load (category, neutral, and sleep conditions). (d) Difference in accuracy between congruent and incongruent conditions in both patients with CFS and controls. (e) Comparisons between incongruent, priming negative inverse, and priming negative simple conditions for accuracy. (f) Difference in accuracy between patients with CFS and controls for sleep-related words, and difference in accuracy between category and sleep stimuli in controls. All comparisons were performed using $3 \times 2$ analysis of variance with condition as within-subject factor and group as between-subject factor. Additional analyses between accuracy scores of both groups in nouns with different levels of emotional load (category, neutral, and sleep conditions) were conducted using Mann-Whitney $U$ test. Error bars indicate standard error. Marginally statistically significant effects: ${ }^{*} p<0.05,{ }^{\dagger} p \leq 0.01,{ }^{\ddagger} p=0.05$. $\mathrm{Cl}=$ confidence interval, $\mathrm{ME}=$ myalgic encephalomyelitis. 
Table 2.

Comparison of psychomotor vigilance task (PVT) and operation span task (OSPAN) scores between patients with chronic fatigue syndrome (CFS) and healthy inactive control participants. Data presented as mean \pm standard deviation. Statistically significant results are bold.

\begin{tabular}{|c|c|c|c|}
\hline Variable & CFS $(n=30)$ & Control $(n=11)$ & $p$-Value \\
\hline \multicolumn{4}{|l|}{$\overline{\mathrm{PVT}}$} \\
\hline Reaction Time (ms) & $357.6 \pm 45.4$ & $289.1 \pm 23.5$ & $<0.001^{*}$ \\
\hline Lapses (\%) & $30.6 \pm 36.6$ & $2.8 \pm 1.9$ & $<0.001^{\dagger}$ \\
\hline Math Error & $7.3 \pm 3.3$ & $6.5 \pm 5.4$ & $0.56^{*}$ \\
\hline Accuracy Error & $5.8 \pm 3.0$ & $3.7 \pm 2.4$ & $0.05^{*}$ \\
\hline Total & $43.6 \pm 22.0$ & $53.5 \pm 16.4$ & $0.19^{*}$ \\
\hline
\end{tabular}

Table 3.

Comparison of physical fitness variables between patients with chronic fatigue syndrome (CFS) and healthy inactive control participants. Data presented as mean \pm standard deviation. Statistically significant results are bold.

\begin{tabular}{lccc}
\hline \multicolumn{1}{c}{ Variable } & CFS $(\boldsymbol{n}=\mathbf{3 0})$ & Control $(\boldsymbol{n}=\mathbf{1 3})$ & $\boldsymbol{p}_{\text {-Value }}$ \\
\hline Resting Heart Rate (bpm) & $92.7 \pm 13.2$ & $97.9 \pm 14.7$ & 0.27 \\
Peak Heart Rate (bpm) & $145.1 \pm 22.4$ & $164.7 \pm 7.0$ & $<\mathbf{0 . 0 0 1}$ \\
Peak Oxygen Uptake (mL/min/kg) & $19.1 \pm 4.6$ & $27.2 \pm 5.6$ & $<\mathbf{0 . 0 0 1}$ \\
Peak Workload (W) & $114.2 \pm 31.3$ & $170.0 \pm 36.2$ & $<\mathbf{0 . 0 0 1}$ \\
Peak Respiratory Exchange Ratio & $1.07 \pm 0.13$ & $1.11 \pm 0.08$ & 0.19 \\
Maximal Handgrip Strength (lbf) ${ }^{\dagger}$ & $50.9 \pm 13.2$ & $58.9 \pm 20.0$ & 0.14 \\
\hline
\end{tabular}

${ }^{*}$ Comparisons performed using Student $t$-test.

${ }^{\dagger}$ Data of only 29 patients with CFS and 12 controls were analyzed due to data loss.

$\mathrm{bpm}=$ beats per minute, $\mathrm{lbf}=$ pound-force.

measured by the PVT and $\operatorname{HR}_{\mathrm{p}}(p<0.05)$. RER $\mathrm{p}$ was associated with the Stroop no word and sleep condition reaction times $(p<0.05)$. Maximal handgrip strength was related to OSPAN accuracy error, score, and total ( $p<$ $0.05)$ but not to OSPAN math error and speed error ( $p>$ 0.05) (Table 4). No other associations between cognitive performance and physical fitness variables were observed $(p>0.05)$.

To determine whether the observed relationships in patients with CFS could not simply be interpreted as normal, we also examined the associations between the same variables in the control group. None of the significant relationships observed in the patients with CFS were found to be significant in the control group $(p>0.05)$.

\section{Relationship Between Cognitive Performance and Physical Activity Level in Patients with Chronic Fatigue Syndrome}

Only two variables measured with the SWA were significantly correlated with cognitive performance. The amount of time that patients slept per day (sleep time) was inversely related to Stroop accuracy for neutral (neutral and no word conditions) and inverse negative priming stimuli $(r=-0.47, r=-0.60$, and $r=-0.49$, respectively; $p<0.05$ ). A negative correlation was found between the number of steps taken per day (steps) and the Stroop accuracy score for incongruent stimuli $(r=-0.53$, $p=0.01)$. The latter correlation was marginally significant in the control group ( $r=-0.63, p=0.05)$. None of the other significant and nonsignificant relationships observed in the patients with CFS were significant in the control group $(p>0.05)$.

\section{DISCUSSION}

The present study aimed at examining the relation between cognitive performance and physical fitness in female patients with CFS. Likewise, the relation between cognitive performance and PAL was examined. In 
Table 4.

Statistically significant associations between cognitive performance and physical fitness variables in patients with chronic fatigue syndrome $(n=26)$.

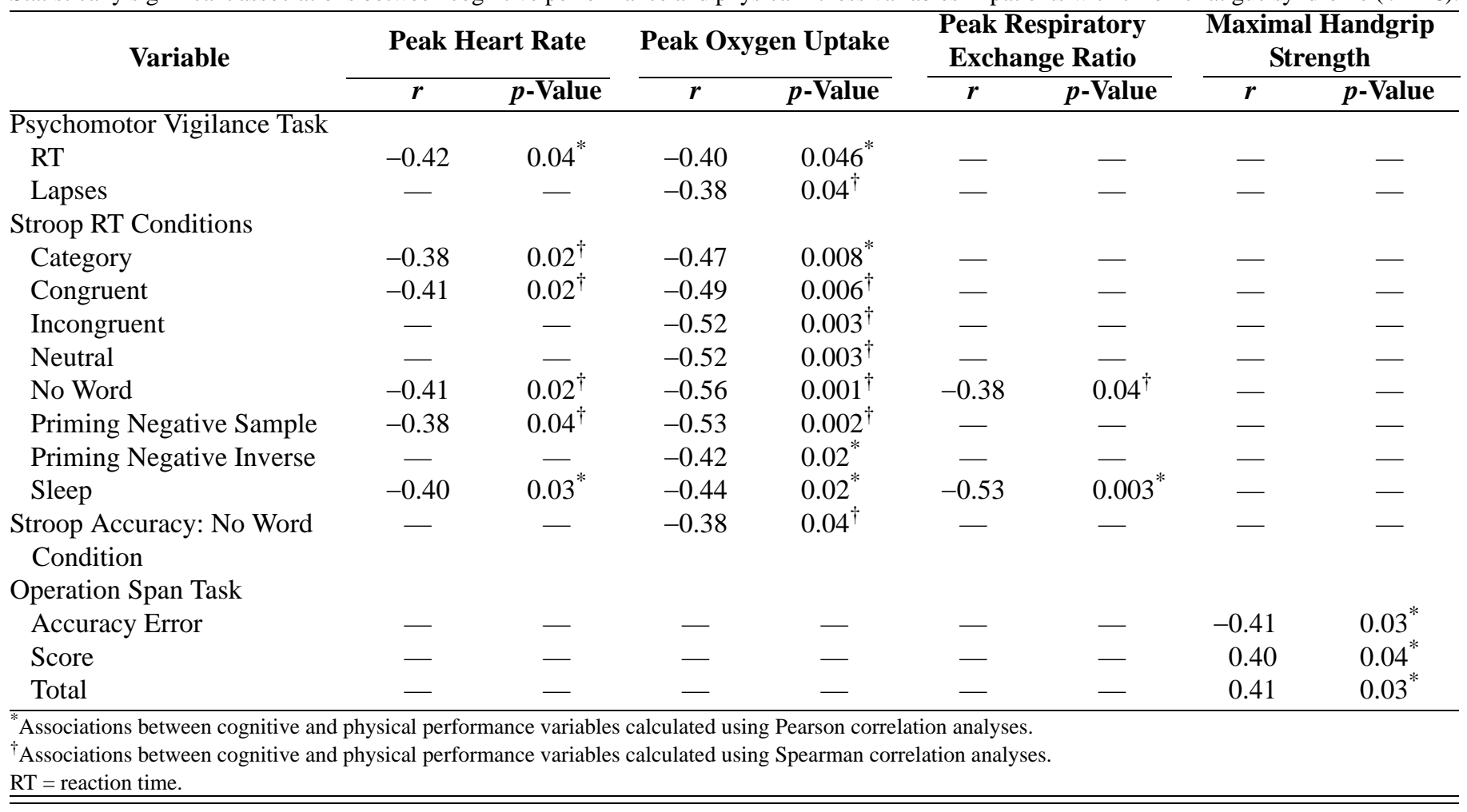

addition, cognitive performance and physical fitness were compared between patients with CFS and healthy controls.

\section{Cognitive Performance}

The results of the present study showed decreased choice and simple reaction time in patients with CFS relative to controls, as demonstrated by slower response times on all conditions of Stroop reaction time and the PVT, respectively. This first finding confirms previous findings also using the Stroop task as an objective measure to evaluate choice reaction times in patients with CFS [8-9]. On the PVT, we found that patients with CFS did not only demonstrate significantly longer reaction times than controls, but they also committed more errors of omission, which resulted in significantly higher lapse scores. The PVT is a test of simple reaction time that is most commonly used in sleep deprivation studies to examine the effect of this phenomenon on attention and vigilance [35-36]. In their literature review, Lim and Dinges state that sleep-deprived persons show an overall slowing of responses and an increase of lapses for lengthy periods on the PVT [36]. In the present study, we have evidence of these findings in patients with CFS as well. Consequently, Lim and Dinges adopt that sleep deprivation suppresses the ability to pay attention [36]. Perceptual, processing, or executive failures in the central nervous system are thought to increase the number of lapses in sleep-deprived persons [36]. This could be a possible explanation for the higher number of lapses determined in patients with CFS. By all means, apart from the degree of sleep deprivation, levels of exhaustion and fatigue should always be taken into account in these patients in future research.

Although we found that overall choice reaction time was lower in patients with CFS relative to controls, as evidenced by slower color naming on all conditions of the Stroop task, Stroop (semantic) interference effect in patients with CFS was not increased relative to controls. Because Stroop interference reflects selective attending ability or the ability to inhibit irrelevant information, this finding demonstrates normal semantic processing in these patients. These findings were not unexpected in that they replicate previous findings [7-9,37-38].

The reaction times of both patients with CFS and controls for the negative priming conditions (simple and 
inverse) compared with the incongruent condition were not significantly different (no negative priming effect). These data therefore show that both patients with CFS and controls are not capable of inhibiting distractor stimuli. To our knowledge, the present study was the first to investigate negative priming effects in patients with CFS. Surprisingly, no negative priming effect was present in controls, which may be the result of our small sample size.

We found no significant emotional interference effect (sleep-related words) in either the patients with CFS or controls. On the other hand, patients with CFS did show shorter reaction times for fatigue-related words than for neutral words. Possibly due to the high variance, this difference was not found to be statistically significant. This suggests that patients with CFS are less attentive to fatigue-related information. These findings are in line with previous reports $[9,39]$, which did not find attentional bias toward illness-related information in individuals with CFS.

Stroop accuracy analyses showed a marginally significant lower score for the incongruent condition than the congruent condition in both groups. This could indicate that no speed-accuracy trade-off took place (patients with CFS and controls did not emphasize speed over accuracy) and consequently could demonstrate that the patients with CFS were really slower in color-naming all conditions relative to the controls. A marginally significant lower score was found in the controls for the sleeprelated words condition than the category condition. Furthermore, patients with CFS were even more accurate than controls in color-naming sleep-related words. These results could suggest that sleep-related words had an emotional load on the controls but not on the patients with CFS. Interestingly, all recruited controls were related in one way or another to the patients with CFS because every patient was asked to bring a healthy and inactive relative, friend, or acquaintance to participate in the control group. Indeed, it has been previously shown that the emotional burden of CFS is felt by carers and relatives as well [40].

Regarding the OSPAN, there are two sources of data: one from the math portion of the task, which is the processing component, and one from the letter span part of the task, which is the storage component. Patients with CFS did not perform significantly worse on either one of the components, but when looking closer at the results, it is remarkable that especially the letter recall scores are worse in the patients with CFS. The reason why there is only a trend and not a significant difference in the letter span scores is the presence of the high variability between the obtained scores, which is in turn likely the result of the presence of one or more confounding variables (e.g., moment of the day the test was taken, levels of education and intelligence, menopausal state, disease duration). Thus, as evidenced several times before [68,11-12], it is likely that patients with CFS have a reduced working memory capacity.

\section{Physical Fitness}

Previous studies show conflicting findings in relation to physiological exercise capacity in patients with CFS [20]. Our findings suggest that female patients with CFS display a decreased exercise capacity, as evidenced by reduced $\mathrm{VO}_{2 \mathrm{p}}$, reduced peak workload, and reduced $\mathrm{HR}_{\mathrm{p}}$. However, values of $\mathrm{HR}_{\text {rest }}$ and $\mathrm{RER}_{\mathrm{p}}$ were found to be near normal. In this study, we anticipated potential sources of bias such as the lack of uniformity in sex, PAL, and utilized diagnostic criteria for CFS. All controls had an inactive lifestyle as defined by De Becker et al. [27], all participants were female, and participating patients with CFS were all diagnosed according to the 1994 CDC criteria to exclude the presence of possible psychiatric disorders.

In a previous study of patients with CFS, Van Oosterwijck et al. demonstrated that postexertional malaise, which is a term used to describe symptom exacerbation as a result of excessive exercise, is triggered by submaximal exercise and self-paced, physiologically limited exercise [41]. Consequently, it is possible to explain the reduced exercise capacity in individuals with CFS by an underlying fear of postexertional malaise. In addition, it is evidenced that patients with CFS report higher ratings of perceived exertion during exercise relative to healthy controls [42-45]. Wallman et al. therefore suggest that the reduced exercise capacity seen in some patients with CFS might be explained by an abnormal sense of effort in these individuals and/or a reluctance to push toward full capacity [45]. On the other hand, we registered a mean $\mathrm{RER}_{\mathrm{p}}$ value of 1.07 in the patients with CFS, which demonstrates that these patients have cycled to the top of their potential. Likewise, it is well documented that patients with CFS exhibit reduced daily PALs [20-21], and (part of) the cause of this deteriorated physical exercise capacity can possibly be found in the entailed downward spiral of physical inactivity and avoidance behavior toward physical activity. 
In this study, we observed no significant difference in maximal muscle (handgrip) strength between individuals with CFS and healthy controls. Although there is controversy in the literature about maximal muscle strength in patients with CFS compared with healthy controls, these results are in line with most studies that examined this parameter in patients with CFS [46-51].

\section{Relationship Between Cognitive Performance and Physical Fitness and Cognitive Performance and Physical Activity Level}

To the best of our knowledge, the present study is the first to address the association between cognitive performance and physical fitness in people with CFS. Our data showed the presence of an association between these two domains in people with CFS. Patients with reduced physiological exercise capacity were more likely to perform worse on cognitive testing. More specifically, those patients with CFS who demonstrated lower $\mathrm{VO}_{2 \mathrm{p}}$ and $\mathrm{HR}_{\mathrm{p}}$ values also showed slower psychomotor speed (longer simple and choice reaction times). Furthermore, moderate correlations were found between maximal handgrip strength and letter recall (positive and negative, respectively) and accuracy errors on the math portion of the OSPAN task.

In contrast with our hypothesis, increased PALs were not associated with better cognitive performance in patients with CFS. We only observed negative relationships between sleep time, steps taken per day, and some Stroop accuracy scores. Our findings are not in line with those of Christodoulou et al. [25], who evidenced a positive relationship between cognitive impairment and functional daily activity disability, and Vercoulen et al. [24], who reported an association between low PALs and slowed motor and information processing speed in persons with CFS.

A posteriori analysis of correlations between physical exercise capacity and PALs showed a positive relationship between these domains in both groups (higher $\mathrm{VO}_{2 \mathrm{p}}$ values were related to higher physical activity time [ $>3$ METs], physical activity energy expenditure [ $>3$ METs], and mean energy expenditure). The fact that lower physical fitness but not lower PALs were associated with poorer cognitive performance, while we also found a significant positive relationship between PAL and $\mathrm{VO}_{2 \mathrm{p}}$, is interesting. As a result of these findings, it can be hypothesized that PAL is a potential mediator of the relationship between $\mathrm{VO}_{2 \mathrm{p}}$ and cognitive function in patients with CFS.

Likewise, Wallman et al. showed an improvement in attentional function after a $12 \mathrm{wk}$ graded exercise intervention [52]. This positive effect of an exercise program has already been observed in healthy people [53] and in other fatigue-related patient populations such as those with multiple sclerosis [54-55], fibromyalgia [56], Parkinson disease [57-58], and cardiac disease [59]. However, in patients with CFS there is still a paucity of objective data concerning this topic. Although weekly energy expenditure was recorded through self-report, results from the randomized controlled trial conducted by Wallman et al. showed an increase in PAL in the graded exercise group [52]. These data strengthen the hypothesis of PAL being a potential mediator of the relationship between $\mathrm{VO}_{2 \mathrm{p}}$ and cognitive function in patients with CFS. This is very important because there still exists an inconclusive discussion around what type of managing program is best suited for this group of patients (cognitive behavioral therapy with graded activity vs graded exercise or pacing). We therefore believe that, in order to shed more light on this discussion, future studies should explore the influence of changes in physical fitness (aerobic capacity), whether or not through changes in physical activity, on cognitive function in patients with CFS. Therefore, continued prospective research (using a randomized controlled design) is warranted.

\section{Study Limitations}

Finally, the present study has a few methodological issues that need to be mentioned. First, caution should be taken when generalizing the results to the complete CFS population, because only women were studied in order to account for bias caused by pooling of sex data. The external validity of the results is therefore limited to adult female patients with CFS who are not housebound. Second, menopausal state of the participants over $40 \mathrm{yr}$ old and levels of education and intelligence were not taken into account. Third, causal interpretation of findings is precluded due to the cross-sectional nature of the study. In addition, longitudinal data are required to examine the stability of these findings in a condition such as CFS, which is characterized by high health status fluctuations over time.

On the other hand, the study also has strengths. The patient group was sufficiently powered, and we anticipated several sources of bias such as medication usage 
and the lack of uniformity in sex, PAL, and utilized diagnostic criteria for CFS. In addition, the measurements used are valid and represent gold standards for measuring physical fitness, cognitive performance, and PAL.

\section{CONCLUSIONS}

In conclusion, the present study confirms the presence of decreased choice and simple reaction time in people with CFS. Moreover, it evidences a significantly decreased physiological exercise capacity, but a preserved maximal handgrip strength in female patients with CFS when compared with healthy inactive controls. Lower levels of physical fitness, but not reduced PALs, were associated with poorer performance on the cognitive testing battery. These observations are important in order to design future studies exploring the influence of changes in physical fitness, whether or not through changes in physical activity, on cognitive function in patients with CFS.

\section{ACKNOWLEDGMENTS}

\author{
Author Contributions: \\ Study concept and design: J. Nijs, L. Lambrecht, M. Meeus, N. Pat- \\ tyn, P. Clarys, E. Zinzen, K. Ickmans. \\ Acquisition of data: K. Ickmans, J. Nijs, S. Aelbrecht, G. Meersdom, \\ D. Aerenhouts. \\ Analysis and interpretation of data: K. Ickmans. \\ Drafting of manuscript: K. Ickmans. \\ Critical revision of manuscript for important intellectual content: \\ P. Clarys, J. Nijs, M. Meeus, D. Aerenhouts, E. Zinzen, S. Aelbrecht, \\ G. Meersdom, L. Lambrecht, N. Pattyn. \\ Statistical analysis: K. Ickmans, N. Pattyn. \\ Administrative, technical, or material support: L. Lambrecht, \\ G. Meersdom, P. Clarys, D. Aerenhouts. \\ Study supervision: J. Nijs, L. Lambrecht. \\ Financial Disclosures: The authors have declared that no competing \\ interests exist. \\ Funding/Support: This material was based on work supported in part \\ by ixX Pharma (Nieuwerken-Waas, Belgium). Ms. Ickmans is a \\ research fellow of ME Research UK (Perth, United Kingdom), a \\ national charity funding biomedical research into myalgic encephalo- \\ myelitis/CFS. Dr. Meeus is a postdoctoral research fellow of the \\ Research Foundation Flanders (Brussels, Belgium). \\ Additional Contributions: The authors would like to thank Tinne \\ Boey and Wouter Rosseels for their aid in the data input. \\ Institutional Review: The study protocol was approved by the Ethics \\ Committee of the University Hospital Brussels/Vrije Universiteit \\ Brussel, and written informed consent was obtained from all study \\ participants.
}

Participant Follow-up: The authors plan to inform participants for whom contact information is available of the publication of this study.

\section{REFERENCES}

1. Fukuda K, Straus SE, Hickie I, Sharpe MC, Dobbins JG, Komaroff A; International Chronic Fatigue Syndrome Study Group. The chronic fatigue syndrome: a comprehensive approach to its definition and study. Ann Intern Med. 1994;121(12):953-59. [PMID:7978722] http://dx.doi.org/10.7326/0003-4819-121-12-199412150-00009

2. Abbey SE, Garfinkel PE. Chronic fatigue syndrome and depression: cause, effect, or covariate. Rev Infect Dis. 1991;13(Suppl 1):S73-83. [PMID:2020805] http://dx.doi.org/10.1093/clinids/13.Supplement 1.S73

3. DeLuca J, Johnson SK, Beldowicz D, Natelson BH. Neuropsychological impairments in chronic fatigue syndrome, multiple sclerosis, and depression. J Neurol Neurosurg Psychiatry. 1995;58(1):38-43. [PMID:7823065] http://dx.doi.org/10.1136/jnnp.58.1.38

4. Komaroff AL, Buchwald DS. Chronic fatigue syndrome: an update. Annu Rev Med. 1998;49:1-13.

[PMID:9509246]

http://dx.doi.org/10.1146/annurev.med.49.1.1

5. Davey NJ, Puri BK, Nowicky AV, Main J, Zaman R. Voluntary motor function in patients with chronic fatigue syndrome. J Psychosom Res. 2001;50(1):17-20.

[PMID:11259796]

http://dx.doi.org/10.1016/S0022-3999(00)00211-7

6. Majer M, Welberg LA, Capuron L, Miller AH, Pagnoni G, Reeves WC. Neuropsychological performance in persons with chronic fatigue syndrome: results from a populationbased study. Psychosom Med. 2008;70(7):829-36. [PMID:18606722] http://dx.doi.org/10.1097/PSY.0b013e31817b9793

7. Marshall PS, Watson D, Steinberg P, Cornblatt B, Peterson $\mathrm{PK}$, Callies A, Schenck CH. An assessment of cognitive function and mood in chronic fatigue syndrome. Biol Psychiatry. 1996;39(3):199-206. [PMID:8837981] http://dx.doi.org/10.1016/0006-3223(95)00131-X

8. Marshall PS, Forstot M, Callies A, Peterson PK, Schenck $\mathrm{CH}$. Cognitive slowing and working memory difficulties in chronic fatigue syndrome. Psychosom Med. 1997;59(1): 58-66. [PMID:9021867]

9. Moss-Morris R, Petrie KJ. Experimental evidence for interpretive but not attention biases towards somatic information in patients with chronic fatigue syndrome. Br J Health Psychol. 2003;8(Pt 2):195-208. [PMID:12804333] http://dx.doi.org/10.1348/135910703321649169

10. Prasher D, Smith A, Findley L. Sensory and cognitive event-related potentials in myalgic encephalomyelitis. 
J Neurol Neurosurg Psychiatry. 1990;53(3):247-53.

[PMID:2324756]

http://dx.doi.org/10.1136/jnnp.53.3.247

11. Capuron L, Welberg L, Heim C, Wagner D, Solomon L, Papanicolaou DA, Craddock RC, Miller AH, Reeves WC. Cognitive dysfunction relates to subjective report of mental fatigue in patients with chronic fatigue syndrome. Neuropsychopharmacology. 2006;31(8):1777-84.

[PMID:16395303]

http://dx.doi.org/10.1038/sj.npp.1301005

12. Joyce E, Blumenthal S, Wessely S. Memory, attention, and executive function in chronic fatigue syndrome. J Neurol Neurosurg Psychiatry. 1996;60(5):495-503. [PMID:8778252] http://dx.doi.org/10.1136/jnnp.60.5.495

13. DeLuca J, Johnson SK, Ellis SP, Natelson BH. Cognitive functioning is impaired in patients with chronic fatigue syndrome devoid of psychiatric disease. J Neurol Neurosurg Psychiatry. 1997;62(2):151-55. [PMID:9048715] http://dx.doi.org/10.1136/jnnp.62.2.151

14. DeLuca J, Johnson SK, Ellis SP, Natelson BH. Sudden vs gradual onset of chronic fatigue syndrome differentiates individuals on cognitive and psychiatric measures. J Psychiatr Res. 1997;31(1):83-90. [PMID:9201650] http://dx.doi.org/10.1016/S0022-3956(96)00052-0

15. Marcel B, Komaroff AL, Fagioli LR, Kornish RJ 2nd, Albert MS. Cognitive deficits in patients with chronic fatigue syndrome. Biol Psychiatry. 1996;40(6):535-41. [PMID:8879474] http://dx.doi.org/10.1016/0006-3223(95)00422-X

16. Fiedler N, Kipen HM, DeLuca J, Kelly-McNeil K, Natelson B. A controlled comparison of multiple chemical sensitivities and chronic fatigue syndrome. Psychosom Med. 1996;58(1):38-49. [PMID:8677287]

17. Grafman J, Schwartz V, Dale JK, Scheffers M, Houser C, Straus SE. Analysis of neuropsychological functioning in patients with chronic fatigue syndrome. J Neurol Neurosurg Psychiatry. 1993;56(6):684-89. [PMID:8509784] http://dx.doi.org/10.1136/jnnp.56.6.684

18. Krupp LB, Sliwinski M, Masur DM, Friedberg F, Coyle PK. Cognitive functioning and depression in patients with chronic fatigue syndrome and multiple sclerosis. Arch Neurol. 1994;51(7):705-10. [PMID:8018045]

http://dx.doi.org/10.1001/archneur.1994.00540190089021

19. Schmaling KB, DiClementi JD, Cullum CM, Jones JF. Cognitive functioning in chronic fatigue syndrome and depression: a preliminary comparison. Psychosom Med. 1994;56(5):383-88. [PMID:7809336]

20. Nijs J, Aelbrecht S, Meeus M, Van Oosterwijck J, Zinzen E, Clarys P. Tired of being inactive: a systematic literature review of physical activity, physiological exercise capacity and muscle strength in patients with chronic fatigue syn- drome. Disabil Rehabil. 2011;33(17-18):1493-1500. [PMID:21166613]

http://dx.doi.org/10.3109/09638288.2010.541543

21. van Weering M, Vollenbroek-Hutten MM, Kotte EM, Hermens HJ. Daily physical activities of patients with chronic pain or fatigue versus asymptomatic controls. A systematic review. Clin Rehabil. 2007;21(11):1007-23.

[PMID:17984153]

http://dx.doi.org/10.1177/0269215507078331

22. Meeus M, van Eupen I, van Baarle E, De Boeck V, Luyckx A, Kos D, Nijs J. Symptom fluctuations and daily physical activity in patients with chronic fatigue syndrome: a casecontrol study. Arch Phys Med Rehabil. 2011;92(11):1820-26. [PMID:22032215]

http://dx.doi.org/10.1016/j.apmr.2011.06.023

23. Wessely S, David A, Butler S, Chalder T. Management of chronic (post-viral) fatigue syndrome. J R Coll Gen Pract. 1989;39(318):26-29. [PMID:2553945]

24. Vercoulen JH, Bazelmans E, Swanink CM, Galama JM, Fennis JF, van der Meer JW, Bleijenberg G. Evaluating neuropsychological impairment in chronic fatigue syndrome. J Clin Exp Neuropsychol. 1998;20(2):144-56. [PMID:9777468] http://dx.doi.org/10.1076/jcen.20.2.144.1160

25. Christodoulou C, DeLuca J, Lange G, Johnson SK, Sisto SA, Korn L, Natelson BH. Relation between neuropsychological impairment and functional disability in patients with chronic fatigue syndrome. J Neurol Neurosurg Psychiatry. 1998;64(4):431-34. [PMID:9576531]

http://dx.doi.org/10.1136/jnnp.64.4.431

26. Sargent C, Scroop GC, Nemeth PM, Burnet RB, Buckley JD. Maximal oxygen uptake and lactate metabolism are normal in chronic fatigue syndrome. Med Sci Sports Exerc. 2002;34(1):51-56. [PMID:11782647] http://dx.doi.org/10.1097/00005768-200201000-00009

27. De Becker P, Roeykens J, Reynders M, McGregor N, De Meirleir K. Exercise capacity in chronic fatigue syndrome. Arch Intern Med. 2000;160(21):3270-77. [PMID:11088089] http://dx.doi.org/10.1001/archinte.160.21.3270

28. Stroop JR. Studies of interference in serial verbal reactions. J Exp Psychol. 1935;18:643-62. http://dx.doi.org/10.1037/h0054651

29. Dinges DF, Powell JW. Microcomputer analyses of performance on a portable, simple visual RT task during sustained operations. Behav Res Meth Instrum Comput. 1985;17(6): 652-55. http://dx.doi.org/10.3758/BF03200977

30. Conway AR, Engle RW. Individual differences in working memory capacity: more evidence for a general capacity theory. Memory. 1996;4(6):577-90. [PMID:8934455] http://dx.doi.org/10.1080/741940997 
31. Peolsson A, Hedlund R, Oberg B. Intra- and inter-tester reliability and reference values for hand strength. J Rehabil Med. 2001;33(1):36-41. [PMID:11480468] http://dx.doi.org/10.1080/165019701300006524

32. Mathiowetz V. Comparison of Rolyan and Jamar dynamometers for measuring grip strength. Occup Ther Int. 2002;9(3):201-9. [PMID:12374997]

http://dx.doi.org/10.1002/oti.165

33. Fruin ML, Rankin JW. Validity of a multi-sensor armband in estimating rest and exercise energy expenditure. Med Sci Sports Exerc. 2004;36(6):1063-69. [PMID:15179178]

34. Johannsen DL, Calabro MA, Stewart J, Franke W, Rood JC, Welk GJ. Accuracy of armband monitors for measuring daily energy expenditure in healthy adults. Med Sci Sports Exerc. 2010;42(11):2134-40. [PMID:20386334] http://dx.doi.org/10.1249/MSS.0b013e3181e0b3ff

35. Lee IS, Bardwell WA, Ancoli-Israel S, Dimsdale JE. Number of lapses during the psychomotor vigilance task as an objective measure of fatigue. J Clin Sleep Med. 2010;6(2):163-68. [PMID:20411694]

36. Lim J, Dinges DF. Sleep deprivation and vigilant attention. Ann N Y Acad Sci. 2008;1129:305-22.

[PMID:18591490] http://dx.doi.org/10.1196/annals.1417.002

37. Moss-Morris R, Petrie KJ, Large RG, Kydd RR. Neuropsychological deficits in chronic fatigue syndrome: artifact or reality? J Neurol Neurosurg Psychiatry. 1996;60(5):474-77. [PMID:8778249]

http://dx.doi.org/10.1136/jnnp.60.5.474

38. Ray C, Phillips L, Weir WR. Quality of attention in chronic fatigue syndrome: subjective reports of everyday attention and cognitive difficulty, and performance on tasks of focused attention. Br J Clin Psychol. 1993;32(Pt 3):357-64. [PMID:8251968]

39. Martin M, Alexeeva I. Mood volatility with rumination but neither attentional nor interpretation biases in chronic fatigue syndrome. Br J Health Psychol. 2010;15(Pt 4):779-96. [PMID:20100398] http://dx.doi.org/10.1348/135910709X480346

40. Nacul LC, Lacerda EM, Campion P, Pheby D, Drachler ML, Leite JC, Poland F, Howe A, Fayyaz S, Molokhia M. The functional status and well being of people with myalgic encephalomyelitis/chronic fatigue syndrome and their carers. BMC Public Health. 2011;11:402.

[PMID:21619607]

http://dx.doi.org/10.1186/1471-2458-11-402

41. Van Oosterwijck J, Nijs J, Meeus M, Lefever I, Huybrechts L, Lambrecht L, Paul L. Pain inhibition and postexertional malaise in myalgic encephalomyelitis/chronic fatigue syndrome: an experimental study. J Intern Med. 2010;268(3): 265-78. [PMID:20412374] http://dx.doi.org/10.1111/j.1365-2796.2010.02228.x
42. Fulcher KY, White PD. Strength and physiological response to exercise in patients with chronic fatigue syndrome. J Neurol Neurosurg Psychiatry. 2000;69(3):302-7. [PMID:10945803] http://dx.doi.org/10.1136/jnnp.69.3.302

43. Riley MS, O’Brien CJ, McCluskey DR, Bell NP, Nicholls DP. Aerobic work capacity in patients with chronic fatigue syndrome. BMJ. 1990;301(6758):953-56. [PMID:2249024]

44. Sisto SA, LaManca J, Cordero DL, Bergen MT, Ellis SP, Drastal S, Boda WL, Tapp WN, Natelson BH. Metabolic and cardiovascular effects of a progressive exercise test in patients with chronic fatigue syndrome. Am J Med. 1996;100(6):634-40. [PMID:8678084] http://dx.doi.org/10.1016/S0002-9343(96)00041-1

45. Wallman KE, Morton AR, Goodman C, Grove R. Physiological responses during a submaximal cycle test in chronic fatigue syndrome. Med Sci Sports Exerc. 2004;36(10): 1682-88. [PMID:15595287] http://dx.doi.org/10.1249/01.MSS.0000142406.79093.90

46. Gibson H, Carroll N, Clague JE, Edwards RH. Exercise performance and fatiguability in patients with chronic fatigue syndrome. J Neurol Neurosurg Psychiatry. 1993; 56(9):993-98. [PMID:8410041] http://dx.doi.org/10.1136/jnnp.56.9.993

47. Samii A, Wassermann EM, Ikoma K, Mercuri B, George MS, O’Fallon A, Dale JK, Straus SE, Hallett M. Decreased postexercise facilitation of motor evoked potentials in patients with chronic fatigue syndrome or depression. Neurology. 1996;47(6):1410-14. [PMID:8960719] http://dx.doi.org/10.1212/WNL.47.6.1410

48. Lloyd AR, Gandevia SC, Hales JP. Muscle performance, voluntary activation, twitch properties and perceived effort in normal subjects and patients with the chronic fatigue syndrome. Brain. 1991;114(Pt 1A):85-98. [PMID:1998892]

49. Lloyd AR, Hales JP, Gandevia SC. Muscle strength, endurance and recovery in the post-infection fatigue syndrome. J Neurol Neurosurg Psychiatry. 1988;51(10):1316-22. [PMID:2852211]

50. Sacco P, Hope PA, Thickbroom GW, Byrnes ML, Mastaglia FL. Corticomotor excitability and perception of effort during sustained exercise in the chronic fatigue syndrome. Clin Neurophysiol. 1999;110(11):1883-91. [PMID:10576483]

51. Rutherford OM, White PD. Human quadriceps strength and fatiguability in patients with post viral fatigue. J Neurol Neurosurg Psychiatry. 1991;54(11):961-64. [PMID:1800667]

52. Wallman KE, Morton AR, Goodman C, Grove R, Guilfoyle AM. Randomised controlled trial of graded exercise in chronic fatigue syndrome. Med J Aust. 2004;180(9): 444-48. [PMID:15115421] 
53. Smith PJ, Blumenthal JA, Hoffman BM, Cooper H, Strauman TA, Welsh-Bohmer K, Browndyke JN, Sherwood A. Aerobic exercise and neurocognitive performance: a metaanalytic review of randomized controlled trials. Psychosom Med. 2010;72(3):239-52. [PMID:20223924] http://dx.doi.org/10.1097/PSY.0b013e3181d14633

54. McDonnell MN, Smith AE, Mackintosh SF. Aerobic exercise to improve cognitive function in adults with neurological disorders: a systematic review. Arch Phys Med Rehabil. 2011;92(7):1044-52. [PMID:21704783] http://dx.doi.org/10.1016/j.apmr.2011.01.021

55. White LJ, Castellano V. Exercise and brain health-implications for multiple sclerosis: Part 1-neuronal growth factors. Sports Med. 2008;38(2):91-100. [PMID:18201113]

56. Munguía-Izquierdo D, Legaz-Arrese A. Assessment of the effects of aquatic therapy on global symptomatology in patients with fibromyalgia syndrome: a randomized controlled trial. Arch Phys Med Rehabil. 2008;89(12):2250-57. [PMID:19061736] http://dx.doi.org/10.1016/j.apmr.2008.03.026

57. Cruise KE, Bucks RS, Loftus AM, Newton RU, Pegoraro R, Thomas MG. Exercise and Parkinson's: benefits for cognition and quality of life. Acta Neurol Scand. 2011;123(1): 13-19. [PMID:20199518] http://dx.doi.org/10.1111/j.1600-0404.2010.01338.x

58. Tanaka K, Quadros AC Jr, Santos RF, Stella F, Gobbi LT, Gobbi S. Benefits of physical exercise on executive func- tions in older people with Parkinson's disease. Brain Cogn. 2009;69(2):435-41. [PMID:19006643]

http://dx.doi.org/10.1016/j.bandc.2008.09.008

59. Carles S Jr, Curnier D, Pathak A, Roncalli J, Bousquet M, Garcia JL, Galinier M, Senard JM. Effects of short-term exercise and exercise training on cognitive function among patients with cardiac disease. J Cardiopulm Rehabil Prev. 2007;27(6):395-99. [PMID:18197075]

Submitted for publication August 24, 2012. Accepted in revised form January 17, 2013.

This article and any supplementary material should be cited as follows:

Ickmans K, Clarys P, Nijs J, Meeus M, Aerenhouts D, Zinzen E, Aelbrecht S, Meersdom G, Lambrecht L, Pattyn $\mathrm{N}$. Association between cognitive performance, physical fitness, and physical activity level in women with chronic fatigue syndrome. J Rehabil Res Dev. 2013;50(6):795810.

http://dx.doi.org/10.1682/JRRD.2012.08.0156

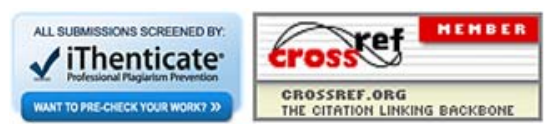


